\title{
Transcriptional regulation of E-cadherin by small activating RNA: A new double-stranded RNA
}

\author{
ZHIMING WU ${ }^{1 *}$, YAN LI $^{2 *}$, ZHIYONG LI ${ }^{1}$, ZHUOWEI LIU $^{1}$, ZIKE QIN $^{1}$, XIANGDONG LI $^{1}$, \\ YUNLIN YE ${ }^{1}$, LEI BU ${ }^{3}$, BIN LIN ${ }^{3}$, ZHANYU WANG ${ }^{2}$, GUOJIN JIA ${ }^{2}$ and GANG CHEN ${ }^{2}$ \\ ${ }^{1}$ Department of Urology, Sun Yat-sen University Cancer Center, State Key Laboratory of Oncology in South China, \\ Collaborative Innovation Center for Cancer Medicine, Guangzhou, Guangdong; \\ ${ }^{2}$ Department of Urology, Jinshan Hospital, Fudan University, Shanghai, P.R. China; \\ ${ }^{3}$ Langone Medical Center School of Medicine, NYU, New York, NY, USA
}

Received April 11, 2016; Accepted July 11, 2016

DOI: $10.3892 /$ ijo.2016.3643

\begin{abstract}
Recent studies have reported that chemically synthesized small activating RNA (saRNA) targeting the promoter regions of a gene can activate its expression in different cell lines. This technique can be a powerful therapeutic method for diseases caused by complete inactivation or reduced expression of specific genes. E-cadherin is a typical tumor suppressor gene. Loss of E-cadherin mediates the transition from benign lesions to invasive, metastatic cancer. In this study, several 21-nt small double-stranded RNAs (dsRNAs) targeting the promoter regions of human E-cadherin were designed and synthesized and the features of their function were investigated to study the regulatory role of dsRNA on E-cadherin expression. A new saRNA (dsEcad-661) that can enhance E-cadherin expression by targeting non-coding regulatory regions in gene promoters was identified. Using dsRNA with modified base quantity and cholesterol-conjugated dsRNA, we found the antisense strand may be the guide strand of saRNA in the upregulation of E-cadherin. These findings provide several important pieces of evidence that may improve understanding of the function of saRNA and may promote its development for clinical application.
\end{abstract}

Correspondence to: Professor Gang Chen, Department of Urology, Jinshan Hospital, Fudan University, 1508 Longhang Road, Shanghai 201508, P.R. China

E-mail: chengangjinshan@163.com

*Contributed equally

Abbreviations: dsRNAs, double-stranded RNAs; saRNA, small activating RNA; RNAa, RNA activation; EMT, epithelial-mesenchymal transition; qPCR, real-time quantitative PCR

Key words: E-cadherin, RNA activation, RNAa, cancer therapy, double-stranded RNAs

\section{Introduction}

Gene silencing by small double-stranded RNA (dsRNA) was first discovered in Caenorhabditis elegans in 1998 (1) and it quickly become an important method of genetic analysis and it has potential in gene therapy. mRNA is generally considered a target for RNA-mediated gene silencing. In the past ten years, studies have shown that dsRNA targeted to gene promoter regions can regulate gene expression at transcription level (2-5). Although the exact mechanisms by which this is regulated are not well understood, these findings demonstrate that dsRNA have more diverse roles in gene regulation than previously recognized. Gene activation by dsRNA is a relatively newly discovered method of inducing gene overexpression $(2,4,6)$. Several studies have shown that some exogenously synthetic dsRNAs can activate a particular gene in a sequence specific manner rather than silencing it $(2,4,7,8)$. This phenomenon has been termed RNA activation (RNAa) and this kind of dsRNA is called small activating RNA (saRNA) (4). Although many mysteries remain, increasing amounts of evidence suggest that RNAa is not only a novel method of studying gene function, but also holds exciting potential for cancer therapeutics (9).

E-cadherin is a cell adhesion molecule. It can mediate cell-cell adhesion and plays a pivotal role in epithelial cell behavior and tissue morphogenesis and remodeling $(10,11)$. Downregulation of E-cadherin is closely associated with tumor invasiveness, metastasis, and poor patient prognosis $(12,13)$. Restoration of E-cadherin by RNAa at the transcriptional level may be a suitable therapy for cancer. Previous studies have shown that some saRNAs can induce E-cadherin gene transcription in a sequence-specific manner $(4,14)$. It is of great interest to identify new saRNA that can upregulate E-cadherin expression efficiently. It is equally important to understand the features and mechanism underlying saRNA function on E-cadherin expression, which can help improve its therapeutic application.

This report describes the design of several new dsRNAs targeted to gene promoter regions and identification of a new saRNA that can significantly enhance E-cadherin expression. The functional strand of the newly discovered dsRNAs targeted to the promoter regions of E-cadherin was also inves- 
Table I. The sequence of synthesized dsRNA and the PCR primer sequence for E-cadherin and GAPDH.

\begin{tabular}{|c|c|c|}
\hline dsRNA name & & Sequence $\left(5^{\prime}-3^{\prime}\right)$ \\
\hline \multirow[t]{2}{*}{ dsE-661 (-661 to -642$)$} & Sense: & CUCAGUGGCUCAUGGCUCA[dT][dT] \\
\hline & Antisense: & UGAGCCAUGAGCCACUGAG[dT][dT] \\
\hline \multirow[t]{2}{*}{ dsE-604 (-604 to -585$)$} & Sense: & GGAUCGCUUCAGCCCAGGA[dT][dT] \\
\hline & Antisense: & UCCUGGGCUGAAGCGAUCC[dT][dT] \\
\hline \multirow[t]{2}{*}{ dsE-454 (-454 to -435$)$} & Sense: & CAGCUACUAGAGAGGCUGG[dT][dT] \\
\hline & Antisense: & CCAGCCUCUCUAGUAGCUG[dT][dT] \\
\hline \multirow[t]{2}{*}{ dsE-382 (-382 to -363$)$} & Sense: & CACCACUGCACUCCAGCUU[dT][dT] \\
\hline & Antisense: & AAGCUGGAGUGCAGUGGUG[dT][dT] \\
\hline \multirow[t]{2}{*}{ dsE-623 (-623 to -604$)$} & Sense: & UUGGGAGGCCAAGGCAGGA[dT][dT] \\
\hline & Antisense: & UCCUGCCUUGGCCUCCCAA[dT][dT] \\
\hline \multirow[t]{2}{*}{ dsE-680 (-680 to -661$)$} & Sense: & CAAAAAAUUAGGCUGCUAG[dT][dT] \\
\hline & Antisense: & CUAGCAGCCUAAUUUUUUG[dT][dT] \\
\hline \multirow[t]{2}{*}{ dsE-648 (-648 to -629$)$} & Sense: & GGCUCACACCUGAAAUCCU[dT][dT] \\
\hline & Antisense: & AGGAUUUCAGGUGUGAGCC[dT][dT] \\
\hline \multirow[t]{2}{*}{ dsControl } & Sense: & UUC UCC GAA CGU GUC ACG U[dT][dT] \\
\hline & Antisense: & ACG UGA CAC GUU CGG AGA A[dT][dT] \\
\hline PCR primer & & Sequence $\left(5^{\prime}-3^{\prime}\right)$ \\
\hline \multirow[t]{2}{*}{ E-cadherin } & Sense: & CGCCGAGAGCTACACGTTCA \\
\hline & Antisense: & TGTCGACCGGTGCAATCTTC \\
\hline \multirow[t]{2}{*}{ GAPDH } & Sense: & CGCTCTCTGCTCCTCCTGTT \\
\hline & Antisense: & CCATGGTGTCTGAGCGATGT \\
\hline
\end{tabular}

tigated. These findings further revealed functional features of saRNA that may be used in mechanistic studies and facilitate its medicinal development for clinical application.

\section{Materials and methods}

dsRNA design and synthesis. Promoter sequence $(1 \mathrm{~kb})$ was picked away from the CpG island and scanned for dsRNA targets based on rational siRNA design rules (9). Four candidates adhered to the rules of functional dsRNA design. Another two saRNA (dsEcad-640 and dsEcad-215) were also synthesized as previously described $(4,14)$. Control dsRNA (dsCon) was specifically designed to have no homology to any known human sequences. Asymmetric dsRNA with 2 or 4 base losses in $5^{\prime}$ ends of either sense or antisense strands were also designed. The asymmetric dsRNA was designed as previously decribed $(15,16)$. All of the dsRNA were chemically synthesized by GenePharma (Shanghai, China) (Table I).

Cell culture and transfection. PC-3 and DU 145 were cultured in RPMI medium-1640 supplemented with 10\% FBS, 2 mM L-glutamine, penicillin $(100 \mathrm{U} / \mathrm{ml})$, and streptomycin $(100 \mu \mathrm{g} / \mathrm{ml})$ in a humidified atmosphere of $5 \% \mathrm{CO}_{2}$ maintained at $37^{\circ} \mathrm{C}$. A hepatocellular carcinoma cell line (MHCC-97L, MHCC-97H, and MHCCLM3) was also cultured using DMEM medium supplemented with $10 \%$ FBS, penicillin (100 U/ml), and streptomycin $(100 \mu \mathrm{g} / \mathrm{ml})$. Transfections of dsRNA were carried out using Lipofectamine 2000
(Invitrogen, USA) according to the manufacturer's protocol and lasted for $72 \mathrm{~h}$.

Real-time quantitative PCR ( $P P C R)$. Total RNA was extracted from cells transfected for $72 \mathrm{~h}$ (mock, $50 \mathrm{nM}$ dsCon, 50 nM dsEcad-661, dsEcad-604, dsEcad-454, dsEcad-382, dsEcad-680, dsEcad-648, dsEcad-623, dsEcad-640 and dsEcad-215). Reverse transcription was performed with a PrimeScript RT Reagent kit (Takara Biotechnology, Dalian, China). qPCR was performed with SYBR Green PCR Reagent kits (Toyobo Co, Osaka, Japan) at a constant annealing temperature $\left(64^{\circ} \mathrm{C}\right)$ according to the manufacturer's protocol. Specific primer sets were designed for qPCR directed against human E-cadherin and GAPDH and synthesized by Takara Biotechnology Co. (Table I). Data were recorded and analyzed using qPCR analysis software Bio-Rad iQ5. Endogenous gene expression was normalized to GAPDH levels in the cells.

Western blot analysis. The expression of E-cadherin protein was estimated using western blotting. Cells were harvested $72 \mathrm{~h}$ after dsRNA treatment as described above and then washed and lysed with M-PER extraction buffer (Pierce Biotechnology) containing protease inhibitors. A BCA assay (Sangon Biotech Co., Ltd., Shanghai, China) was used to measure the concentration of protein lysates. Protein was separated on reducing SDS-polyacrylamide gels and transferred to polyvinyl difluoride membranes (PVDF, Millipore). The membranes were blocked with $3 \%$ BSA buffer for $1 \mathrm{~h}$ 
A

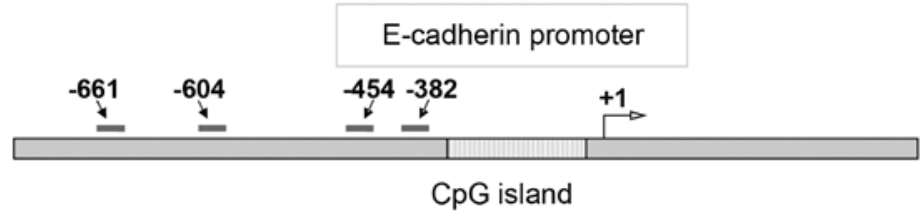

B
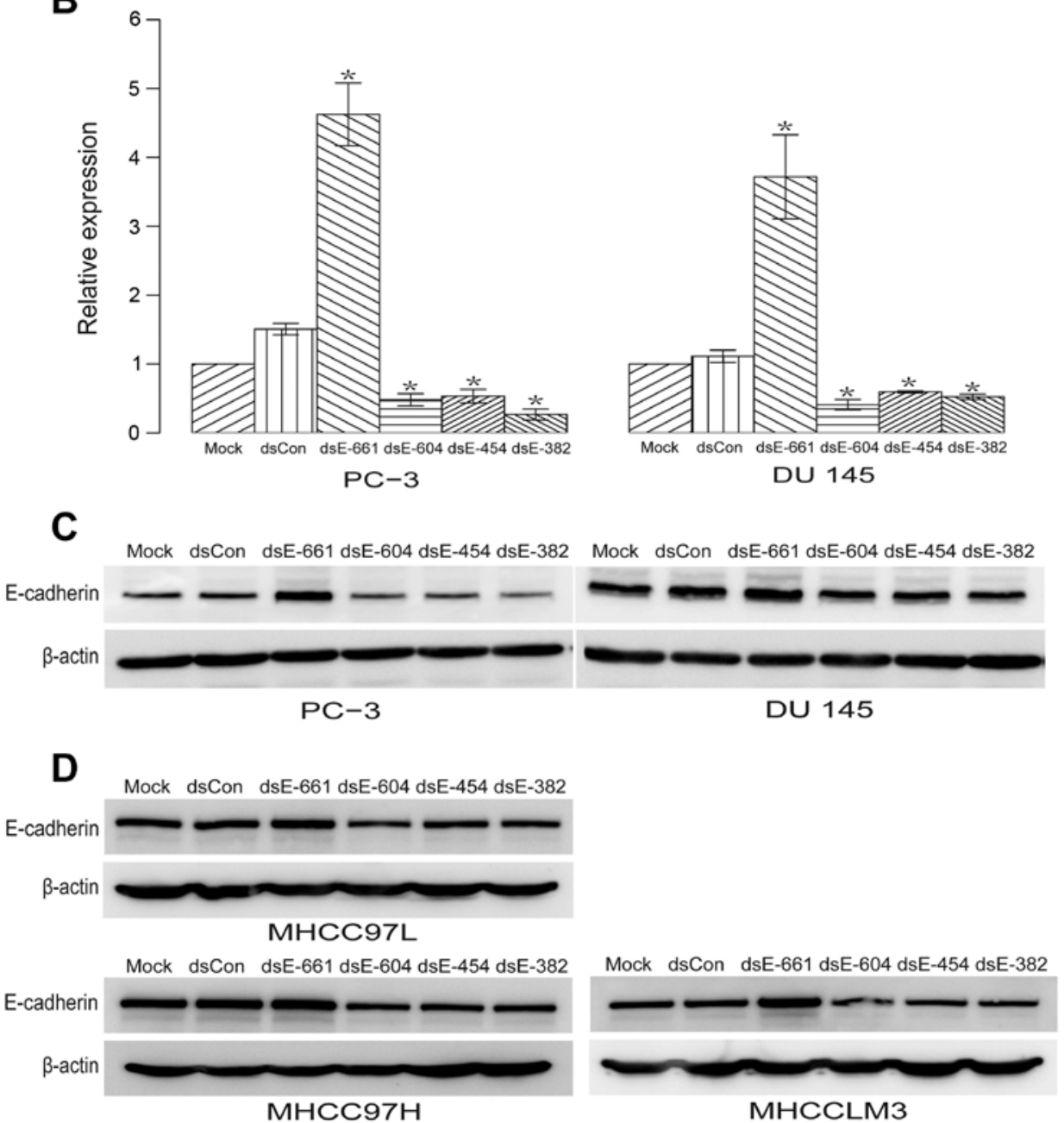

Figure 1. Effects of four dsRNA candidates on E-cadherin mRNA and protein expression in prostate cancer cell lines (PC-3 and DU 145) and HCC cell lines (MHCC97L, MHCC97H and HCCLM3). (A) A schematic diagram showing the target sequence positions of dsRNA candidate (dsEcad-661, dsEcad-604, dsEcad-454 and dsEcad-382). (B) Induction of E-cadherin mRNA expression was measured by qPCR. mRNA expression levels were normalized to GAPDH and presented as means \pm SD of three independent experiments. (C) E-cadherin and $\beta$-actin protein levels were detected by western blot analysis at $72 \mathrm{~h}$ after dsRNAs treatment. (D) Regulation of E-cadherin protein expression by dsRNA candidate in HCC cell lines of MHCC97L, MHCC97H and HCCLM3.

at room temperature and incubated with primary antibodies (anti-E-cadherin rabbit antibody, Cell Signaling, Beverly, MA, USA, 1:1,000 dilutions; anti- $\beta$-actin antibody, Cell Signaling, 1:1,000 dilutions) overnight at $4^{\circ} \mathrm{C} . \beta$-actin levels were used to normalize loading. Then the membranes were incubated with goat anti-rabbit secondary antibody (Cell Signaling) 1:5,000 at $25^{\circ} \mathrm{C}$ for $2 \mathrm{~h}$. Antigen-antibody complexes were visualized using an enhanced chemiluminescence detection method (ECL kit, Thermo, Waltham, MA, USA).

Fluorescence microscopy analysis. Labeling of dsRNAs by Cy3 was performed by Guangzhou RiboBio Co. (Guangzhou, China). Cells were fixed with paraformaldehyde (PFA) at $6 \mathrm{~h}$ after dsRNAs treatment. Staining of the DNA with 4', 6-diamidin-2'-phenylindol-dihydrochlorid (DAPI) was conducted after fixation. A Leica TCS SP5 confocal laser scanning microscope was used for fluorescence imaging.

Statistical analysis. All experiments were performed at least three times. Statistical analysis of all data was performed using Student's t-test. A P-value $<0.05$ was considered to indicate statistical significance.

\section{Results}

New dsRNA targeting the promoter and enhancement of E-cadherin expression. Four 21-nt dsRNAs targeting the E-cadherin promoter at sequence positions -661 (dsEcad-661), -604 (dsEcad-604), -454 (dsEcad-454), and -382 (dsEcad-382) were designed and synthesized (Fig. 1A). Regions with either 


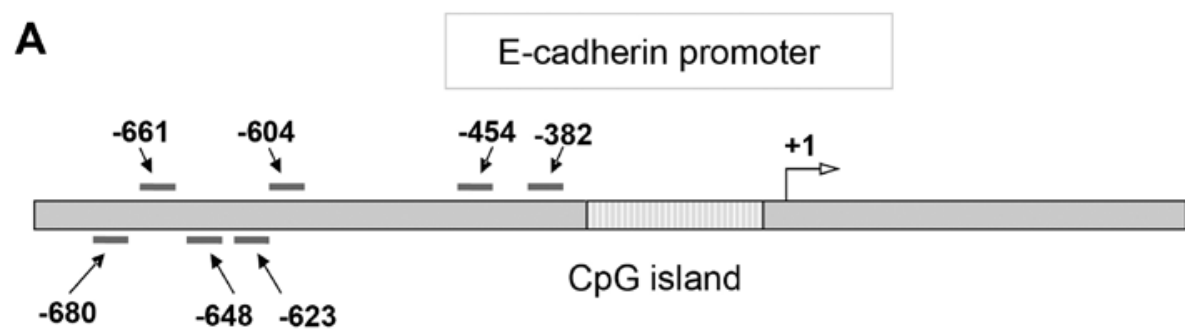

B
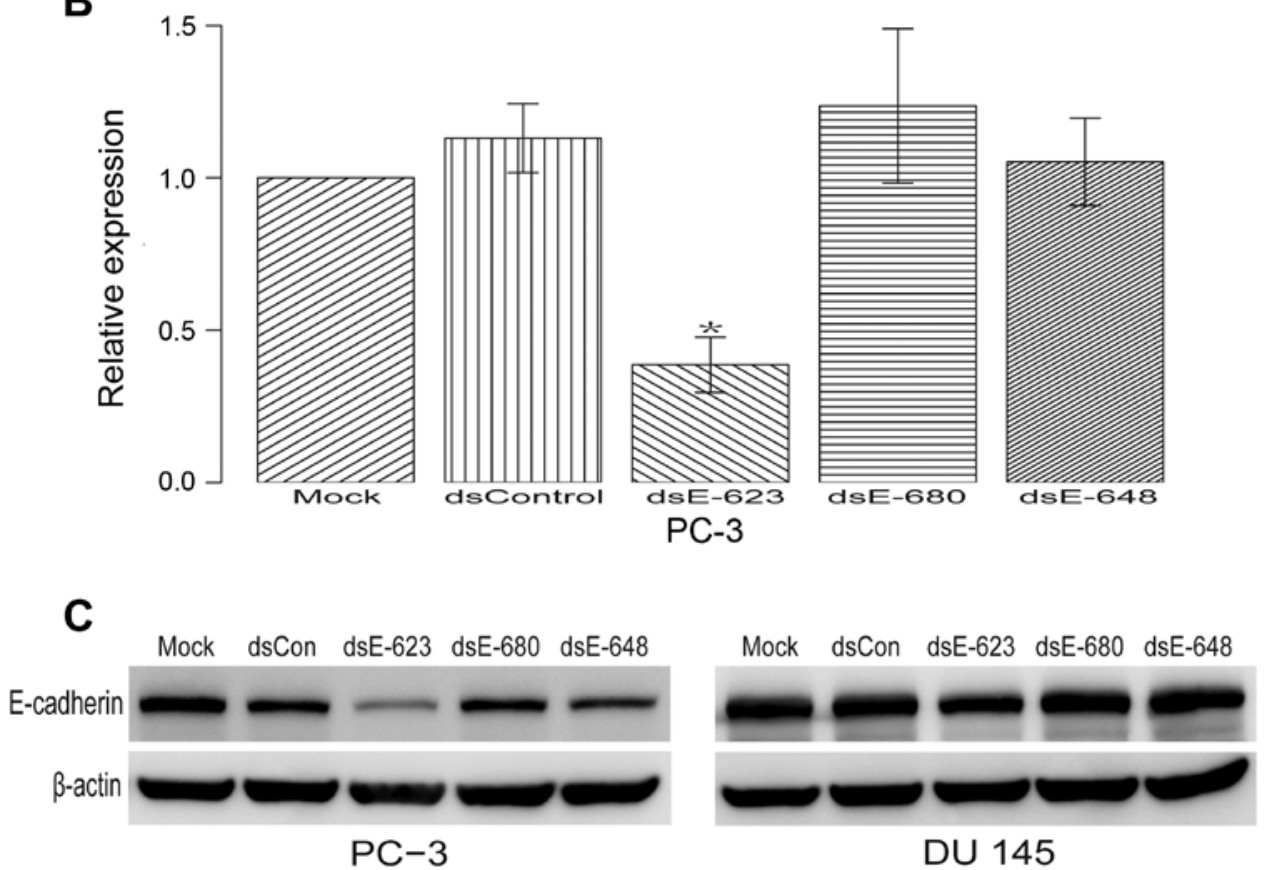

Figure 2. Effect of dsEcad-680, dsEcad-648 and dsEcad-623 on E-cadherin mRNA and protein expression. (A) Three new targeting sequence positions close to the targeting position of dsEcad-661 and dsEcad-640. (B) PC-3 cells and DU 145 cells were transfected with dsRNA for $72 \mathrm{~h}$. mRNA expression of E-cadherin and GAPDH were analyzed by qPCR. (C) Protein expression of E-cadherin was measured by western blot analysis. dsEcad-623 can reduce the expression of E-cadherin while dsEcad-680 and dsEcad-648 has no regulatory activity.

high GC content or low sequence complexity were excluded as dsRNA targets. These included the $\mathrm{CpG}$ island and the Alu repeat element in the E-cadherin promoter (Fig. 1A). A 21-nt dsCon without significant homology to any known human sequence was also synthesized as described previously (4). These dsRNAs were transfected into two human prostate cancer cell lines: PC-3 and DU 145. The expression of E-cadherin mRNA and protein was evaluated using qPCR and western blotting, respectively. We observed a profound induction of E-cadherin mRNA (Fig. 1B) and protein expression (Fig. 1C) by dsEcad-661. While dsEcad-604, dsEcad-454 and dsEcad-382 significantly reduce the expression of E-cadherin mRNA and protein (Fig. 1B and C). To determine whether these effect of dsRNAs are specific to prostate cancer cell lines, these dsRNAs were transfected into hepatocellular carcinoma cell line MHCC-97L, MHCC-97H, and MHCCLM3. The results showed that these dsRNAs have similar regulatory effects on the expression of E-cadherin protein in MHCC-97L, MHCC-97H, and MHCCLM3 cell lines (Fig. 1D). Overall, no changes in E-cadherin expression were detected in cells transfected with mock or dsCon (Fig. 1B-D).

Previous studies have shown that a $21-n t$ dsRNA targeting the E-cadherin promoter at sequence positions -640
(dsEcad-640) can also induce E-cadherin expression (14). To further confirm whether there is any other target near the position -640 and -661 that can also induce the expression of E-cadherin, another three dsRNAs (dsEcad-680, dsEcad-648 and dsEcad-623) were tested. The results indicated that dsEcad-623 can significantly decrease E-cadherin expression. Neither dsEcad-680 nor dsEcad-648 was capable of regulating E-cadherin expression (Fig. 2B and C).

Identification of guide strand. Although the RNAa phenomenon was identified almost a decade ago, the precise mechanism underlying the function still remains unclear. There is some question regarding the saRNA targets: DNA or non-coding RNA transcripts (17-19). Growing evidence suggests that only one strand may be required to guide activity of the dsRNA (20). To identify the target of saRNA, which strand of saRNA guides gene activation must first be determined. Hence, asymmetric saRNA molecules were synthesized without 2 or 4 base in the $5^{\prime}$ end of either the sense or antisense strand, which has been reported to have fewer off-target effects (15). dsEcad-661 (17/19), dsEcad-661(19/17), dsEcad-661(15/21), and dsEcad-661(21/15) were transfected to PC-3 and DU 145 cell line and their activity was analyzed, respectively, using 
A

Symmetric dsRNA: dsE-661 S: 5'-3' asymmetry dsRNA $17 / 19$ (S/AS): asE-661 S: $5^{\prime}-3$ asE-661 AS: 3 $19 / 17$ (S/AS)

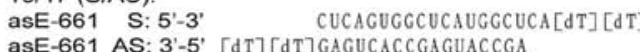
asE-661 AS: $3^{\prime}-5^{\prime}$ [dT] [dT] GAGUCACCGAGUACCG. 15/21(S/AS): asE-661 S: 5'-3' asE-661 AS: $3^{\prime}-5$ 21/15(S/AS): asE-661 S: $5^{\prime}-3^{\prime}$ asE-661 AS: $3^{\prime}-5^{\prime}$

\section{AGUGGCUCAUGGCUC} GAGUCACCGAGUACCGAGU AA

CUCAgUgGCUCAUGgCUCA UU UCACCGAGUACCGAG

\section{S-Chol-modified dsE-661:}

Cholesterol

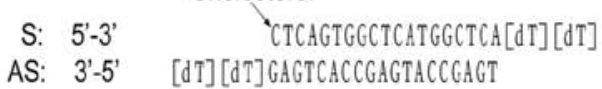

AS-Chol-modified dsE-661:

S: $5^{\prime}-3^{\prime}$

CTCAGTGGCTChTGGCTCA[dT] [dT]

AS: $3^{\prime}-5^{\prime} \quad$ [dT][dT]GAGTCACCGAGTACCGAGT,

Cholesterol
B

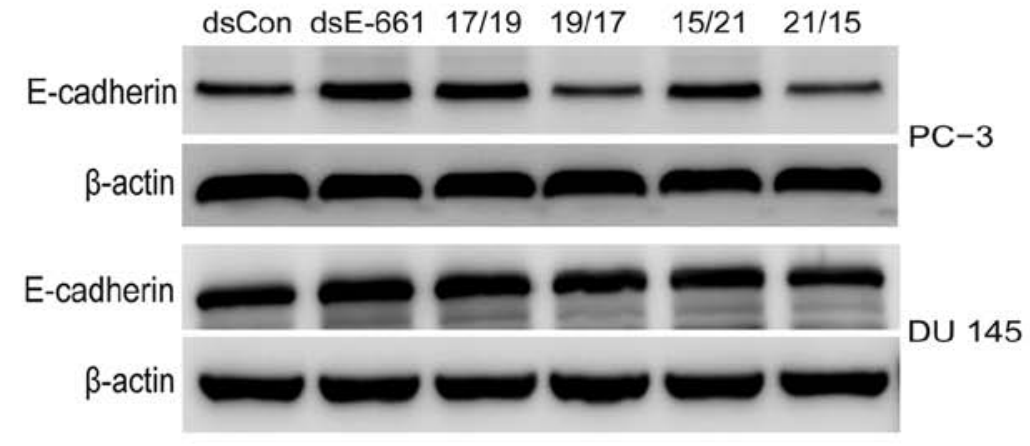

D

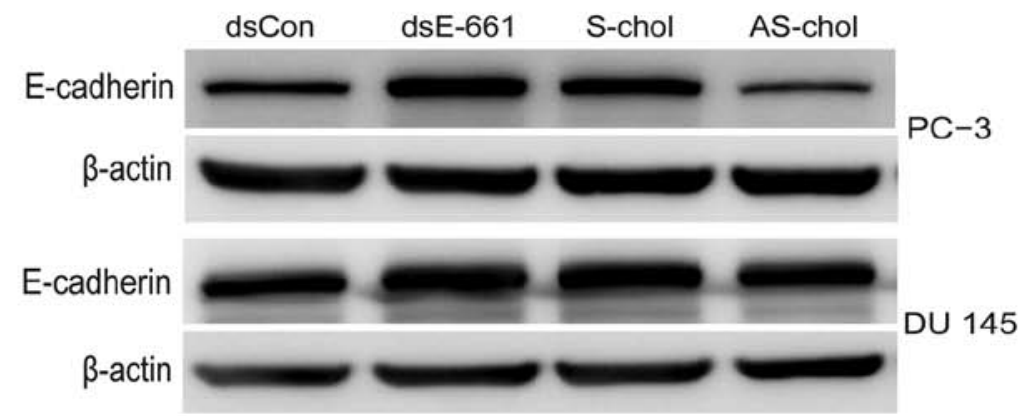

Figure 3. Transfection of modified saRNA indicates that antisense of the saRNA is the guide strand. (A) The sequence of symmetric dsEcad-661 and asymmetric dsEcad-661 molecules with 2 or 4 base losses in either sense or antisense. (B) Protein expression of E-cadherin was measured by western blot analysis after transfection of the symmetric dsEcad-661 and asymmetric dsEcad-661. (C) Antisense stand of dsEcad-661 and the sense strand of dsEcad-661 were linked by with cholesterol respectively. (D) Protein expression of E-cadherin was measured by western blot analysis after transfection of cholesterol-modified dsEcad-661.

western blot analysis. The results showed asymmetric dsEcad-661(17/19) and dsEcad-661(15/21) to have the same E-cadherin activating ability as symmetric dsEcad-661 while asymmetric dsEcad-661(19/17) and dsEcad-661(21/15) lost their gene induction activity (Fig. 3). Another two E-cadherin saRNA (dsE-640 and dsE-215) were also tested as described previously $(4,14)$. The asymmetric dsEcad-640(17/19) and dsE-215(17/19) can induce E-cadherin expression while dsEcad-640(19/17) and dsEcad-215(19/17) lost gene regulating activity (Fig. 4). This evidence indicated that the antisense strand in saRNA duplexes was responsible for RNAa activity. The target of saRNA therefore is expected to be in the sense strand of E-cadherin DNA or the corresponding non-coding RNA transcript.

To further confirm whether the antisense strand is the guide strand of saRNA, the antisense stand of dsEcad-661 and the sense strand of dsEcad-661 was modified with cholesterol respectively, and transfected into PC-3 and DU. The cholesterolconjugated saRNA at the sense stand of dsEcad-661 showed similar gene induction activity while cholesterol-conjugated saRNA at the antisense stand of dsEcad-661 did not show any regulatory effect on E-cadherin expression (Fig. 3).

To determine whether the promoter targeting dsRNA that can downregulate E-cadherin expression share the same target strand as saRNA, another four asymmetric dsRNA were synthesized: dsEcad-604 (17/19), dsEcad-604 (15/21),
dsEcad-604 (19/17), and dsEcad-604 (21/15). The symmetric dsEcad-604 (21/21) and asymmetric dsEcad-604 were transfected into PC-3 and DU 145 cell lines as described previously. Results showed that dsEcad-604 (19/17) and dsEcad-604 $(21 / 15)$ had gene silencing activity similar to that of symmetric dsEcad-604 while dsEcad-604 (17/19) and dsEcad-604 (15/21) lost gene silencing activity (Fig. 5). These results indicated that the sense strand of interfering dsRNA might be the guide strand.

Identification of nucleus entering strand. Previous studies have shown that small interfering dsRNA may, in addition to eliminating specific pools of mRNA in the cytoplasm, also work by epigenetic regulation of gene expression in the nucleus $(21,22)$. To develop a preliminary understanding of the position where saRNA exerts its effects and confirm which strand of saRNA enters the nucleus, the sense and antisense strands of dsEcad-661 were labeled with $\mathrm{Cy} 3$ and transfected using the procedure described above. Sense and antisense strands of dsCon served as the control group. Immunofluorescence analysis indicated that both sense and antisense strands of dsEcad-661 were present in the cytoplasm and nucleus, which indicated that both strands of dsEcad-661 can enter the nucleus (Fig. 6). The control dsRNA without homology to any known human sequence can also enter the nucleus of PC-3 and DU 145 cell (Fig. 6). 
A

Symmetric dsRNA: dsE-215 S: $5^{\prime}-3^{\prime}$ dsE-215 AS: $3^{\prime}-5^{\prime}$ [dT] [dT]UUGGCACGUCCAGGGUAUU

asymmetry dsRNA

$17 / 19$ (S/AS) :

asE-215 S: 5'-3'

asE-215 AS: $3^{\prime}-5^{\prime}$ [d T] [dT]UUGGCACGUGCCCAUAA [dT] [dT]

19/17 (S/AS):

asE-215 S: 5'-3' AACCGUGCAGGUCCCAUAA [dT] [dT]

asE-215 AS: 3'-5' [dT] [dT]UUGGCACGUCCAGGGUA

\section{C}

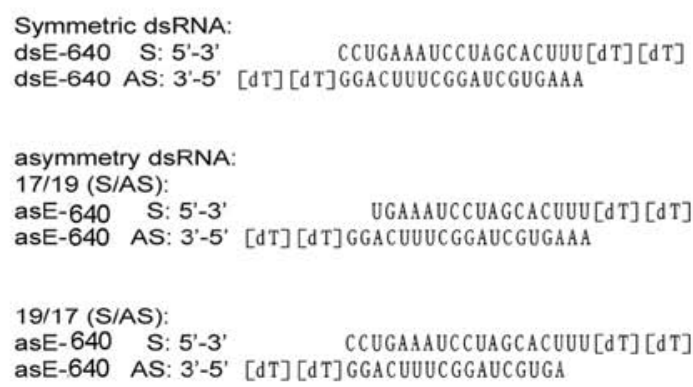

D

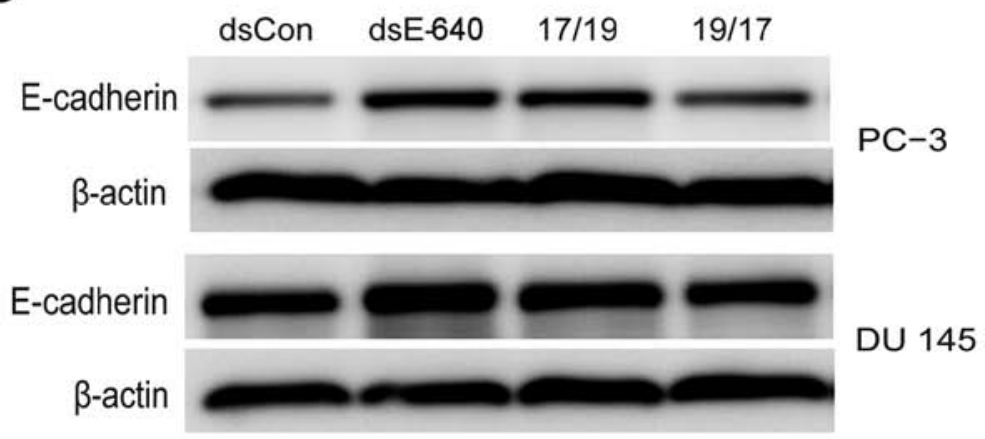

Figure 4. Transfection of modified saRNA (dsEcad-215 and dsEcad-640) indicates that antisense of the saRNA is the guide strand. (A) The sequence of symmetric dsE-215 and asymmetric dsEcad-215 molecules. (B) Protein expression of E-cadherin was measured by western blot analysis after transfection of the symmetric dsEcad-215 and asymmetric dsEcad-215. (C) The sequence of symmetric dsEcad-640 and asymmetric dsEcad-640. (D) Protein expression of E-cadherin was measured by western blot analysis after transfection of the symmetric dsEcad-640 and asymmetric dsEcad-640.

A ymmetric dsRNA:

dsE-604 S: 5'-3' dsE-604 AS: 3'-5' [dT] [dT] CCUAGCGAAGUCGGGUCCU

asymmetry dsRNA:

$17 / 19$ (S/AS):

asE-604 S: 5'-3' AUCGCUUCAGCCCAGGA [d T] [d T]

asE-604 AS: 3'-5' [d T] [d T] C CUAGCGAAGUCGGGUCCU

19/17 (S/AS):

asE-604 S: 5'-3' GGAUCGCUUCAGCCCAGGA [d T] [d T]

asE-604 AS: 3'-5' [d T] [d T] CCUAGCGAAGUCGGGUC

15/21(S/AS):

asE-604 S: 5'-3' UCGCUUCAGCCCAGG

asE-604 AS: 3'-5' CCUAGCGAAGUCGGGUCCU AA

21/15(S/AS):

asE-604 S: 5'-3' GGAUCGCUUCAGCCCAGGA UU

asE-604 AS: 3'-5' AGCGAAGUCGGGUC

B

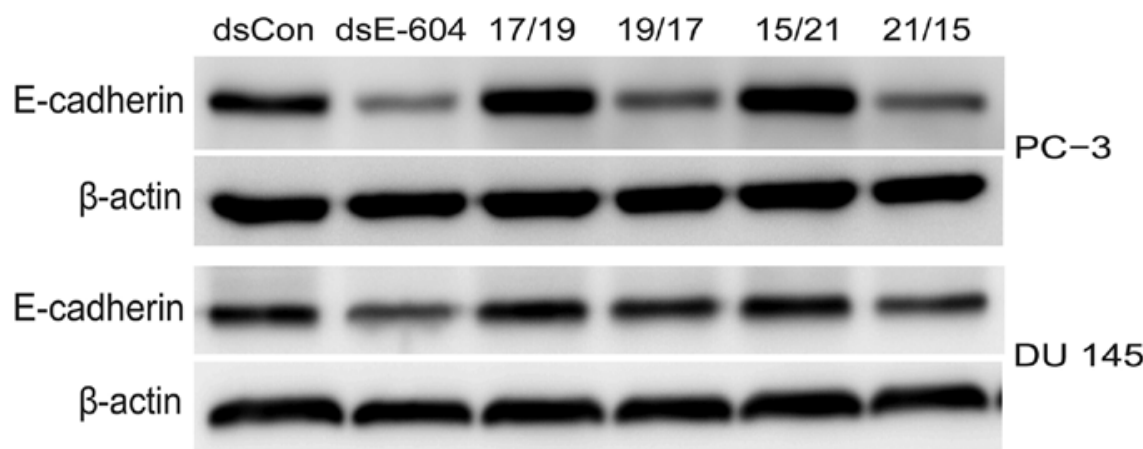

Figure 5. Transfection of modified dsRNA (dsEcad-604) indicates that sense of the dsRNA is the guide strand. (A) The sequence of symmetric dsEcad-604 and asymmetric dsEcad-604 molecules. (B) Protein expression of Ecad-cadherin was measured by western blot analysis after transfection of the symmetric dsEcad-604 and asymmetric dsEcad-604. 

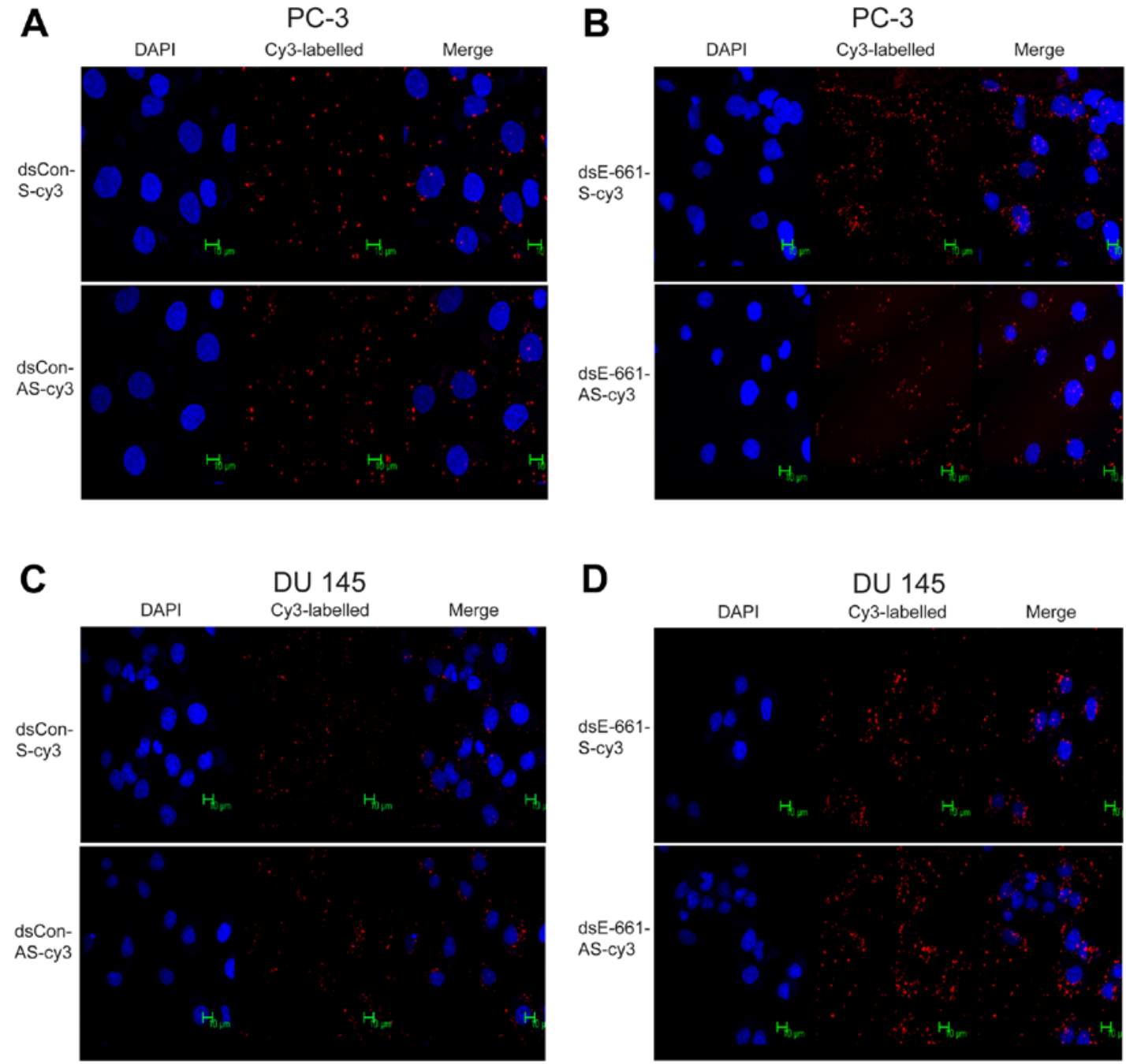

Figure 6. The location of cy3-labeled dsEcad-661 and dsCon in PC-3 and DU 145 cells. (A) The sense and antisense strand of dsCon was labeled by cy3 respectively. PC-3 cells were transfected with cy3-labeled dsCon and then analyzed by fluorescence microscopy. (B) The sense and antisense strand of dsEcad-661 was labeled by cy3 respectively. PC-3 cells were transfected with cy3-labeled dscon and then analyzed by fluorescence microscopy. (C) The sense and antisense strand of dsCon was labeled by cy3 respectively. DU 145 cells were transfected with cy3-labeled dsCon and then analyzed by fluorescence microscopy. (D) The sense and antisense strand of dsEcad-661 was labeled by cy3 respectively. DU 145 cells were transfected with cy3-labeled dscon and then analyzed by fluorescence microscopy.

\section{Discussion}

Downregulated E-cadherin is a key event during epithelialmesenchymal transition (EMT) and is associated with enhanced tumor invasion and poor prognosis in variety of solid tumors (23-25). RNAa is a recently discovered mechanism of gene regulation. It is mediated by dsRNA that targets gene regulatory sequences. saRNA can activate the downregulation of gene expression and so provide a new therapeutic tool for treatment of cancer. In this study, several potential dsRNAs targeting the promoter sequence of E-cadherin were designed and a new saRNA that can induce significant E-cadherin expression was discovered. Previous studies have reported three saRNA (dsEcad-302, dsEcad-215 and dsEcad-640) can induce E-cadherin expression $(4,14)$. These results and the newly discovered dsEcad-661 suggest that E-cadherin expression can be activated by a wide array of saRNA targeting the promoter sequence. saRNA targeting the E-cadherin promoter may serve as a useful tool for further elucidation of the roles of
E-cadherin in cancer and make it possible to develop new gene therapies for cancer. In this study, several dsRNAs targeting the promoter area close to the target of saRNA were found to downregulate E-cadherin expression. These findings suggest that dsRNA have more diverse roles in gene regulation than previously thought. The mechanism by which dsRNA targets the promoter regions and so mediates both gene silencing and gene activation may be very complicated.

Although the RNAa phenomenon has been known for almost a decade, the use of this technique as a therapeutic method has not yet been widely used. Poor understanding of the mechanisms by which it exerts its effects may be the main reason (7). One of these unclear mechanisms is the manner in which synthesized saRNA enters the nucleus and exerts its effect. Previous studies have demonstrated that exogenous dsRNAs may be processed by removing one strand of dsRNA to form active argonaute protein-RNA complexes containing a selected guide strand RNA and then enter the nucleus (26-28). The active complex is guided to its target region by the guide 
A

\section{Gene activation}

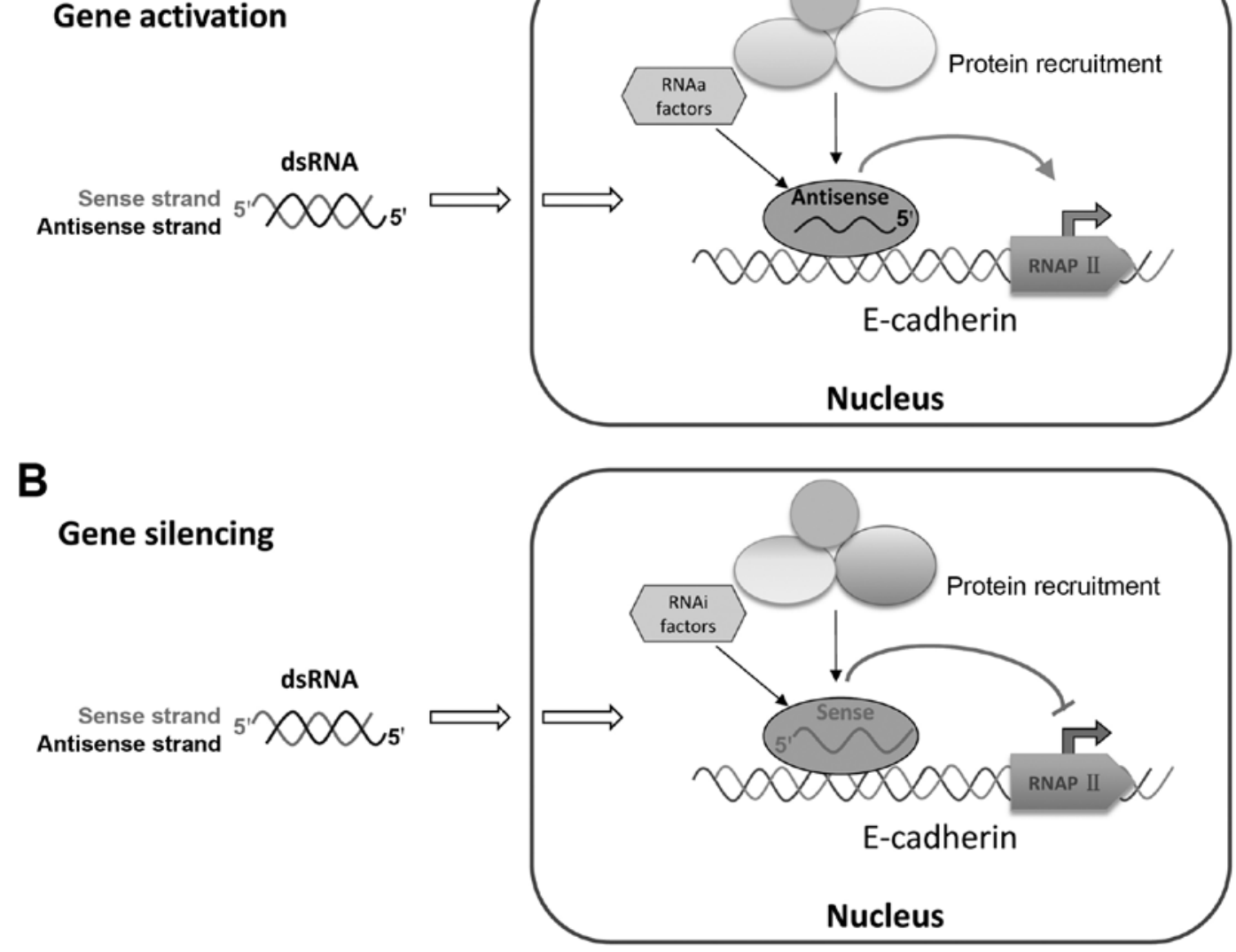

Figure 7. Working model for activating dsRNA and silencing dsRNA. Evidence suggests that antisense strand is required to guide transcriptional gene activation and the sense strand serves as a docking site for target recognition during transcriptional gene silencing.

strand, which may bind to complementary targets on either promoter DNA or nascent promoter RNA $(9,27)$. A recent study also found the mechanism that saRNA-loaded Argonaut protein 2 facilitates the assembly of an RNA-induced transcriptional activation (RITA) complex, which interacts with RNA polymerase II to stimulate transcription initiation and productive elongation (29). In this study, fluorescently labeled dsRNA was used as a direct indicator of which strand can enter the nucleus. Results showed that both strands of dsRNA can enter the nucleus, which suggests that the unwinding process may take place after the dsRNA enters the nucleus. The active argonaute protein-RNA complex may form inside the nucleus. Further studies on this mechanism seem warranted.

It is still not clear how saRNAs activate genes. The activation mechanism of RNAa may involve both transcriptional and epigenetic alterations. The target of saRNA may be either promoter DNA or nascent promoter RNA transcript $(18,19)$. It is also not clear which strand guides RNAa activity when targeting DNA or nascent promoter. Recent studies have provided evidence that RNAa activity was achieved by its antisense strand with the 5 ' region playing a pivotal role (30). However, there still are controversies on the functional strands of saRNA. Some reports have suggested that the sense strand plays a pivotal role (18). As such, we designed and synthesized several asymmetric dsRNA including our newly discovered saRNA (dsEcad-640), previously described saRNA (dsEcad-640 and dsEcad-215) and interfering dsRNA
(dsEcad-604). Using asymmetric dsRNA which has one inactivated strand, we showed that the antisense strand in saRNA duplexes was responsible for the RNAa activity of E-cadherin while the sense strand of interfering dsRNA was responsible for E-cadherin gene silence. To further confirm our results, we designed the cholesterol-conjugated saRNA to block the binding function of each strand selectively. The result also demonstrated that the antisense strand of saRNA was responsible for RNAa activity. This result suggested that the targets of activating dsRNA and silencing dsRNA are located on different strands of DNA or nascent promoter RNA. Previous studies on RNA-directed transcriptional gene regulation have provided a working model for the mechanism of action, which requires the recruitment of several proteins (argonaute-1 and argonaute-2), small RNAs and other factors (31-34). This study brought up fresh evidence that the antisense strand of the activating dsRNA is responsible for RNAa activity and that the sense strand in silencing dsRNA guides gene activation (Fig. 7).

Recent studies showed the gene silencing mediated by asymmetric small interfering RNA is efficacious, durable, and associated with reduced off-target silencing by the sense strand (15). Asymmetric small interfering RNA structure has several advantages over the conventional 19+2 siRNA structures $(16,35)$. This study found that asymmetric saRNA also has gene activating capacity similar to that of conventional $19+2$ siRNA structures. It provides a new structural scaffold 
for designing activating RNA duplex and may facilitate RNAa applications in functional genomics and therapeutics.

RNAa offers a promising new therapeutic strategy for the treatment of diseases with abnormal gene downregulation. Currently, developing RNAa technology against diseases is still at the initial stage. Many of the challenges facing RNA interference based strategies are also applicable to RNAa. Further clarifying the mechanism of the saRNA action is critical for the clinical application of saRNA. In conclusion, a new target of dsRNA was here found to enhance E-cadherin expression and several new dsRNA targets that can downregulate E-cadherin expression. This study also established which strand of saRNA is the guide strand. Further studies are still needed to gain insight into the details of the mechanisms and biology of RNAa.

\section{Acknowledgements}

This study was supported by Shanghai Municipal Commission of Health and family planning (no. 201540372).

\section{References}

1. Fire A, Xu S, Montgomery MK, Kostas SA, Driver SE and Mello CC: Potent and specific genetic interference by doublestranded RNA in Caenorhabditis elegans. Nature 391: 806-811, 1998.

2. Janowski BA, Younger ST, Hardy DB, Ram R, Huffman KE and Corey DR: Activating gene expression in mammalian cells with promoter-targeted duplex RNAs. Nat Chem Biol 3: 166-173, 2007.

3. Elbashir SM, Harborth J, Lendeckel W, Yalcin A, Weber K and Tuschl T: Duplexes of 21-nucleotide RNAs mediate RNA interference in cultured mammalian cells. Nature 411: 494-498, 2001.

4. Li LC, Okino ST, Zhao H, Pookot D, Place RF, Urakami S, Enokida $\mathrm{H}$ and Dahiya R: Small dsRNAs induce transcriptional activation in human cells. Proc Natl Acad Sci USA 103: 17337-17342, 2006.

5. Ting AH, Schuebel KE, Herman JG and Baylin SB: Short doublestranded RNA induces transcriptional gene silencing in human cancer cells in the absence of DNA methylation. Nat Genet 37: 906-910, 2005.

6. Place RF, Noonan EJ, Földes-Papp Z and Li LC: Defining features and exploring chemical modifications to manipulate RNAa activity. Curr Pharm Biotechnol 11: 518-526, 2010.

7. Pushparaj PN, Aarthi JJ, Kumar SD and Manikandan J: RNAi and RNAa - the yin and yang of RNAome. Bioinformation 2: 235-237, 2008.

8. Wang T, Li M, Yuan H, Zhan Y, Xu H, Wang S, Yang W, Liu J, Ye Z and Li LC: saRNA guided iNOS up-regulation improves erectile function of diabetic rats. J Urol 190: 790-798, 2013.

9. Zheng L, Wang L, Gan J and Zhang H: RNA activation: Promise as a new weapon against cancer. Cancer Lett 355: 18-24, 2014.

10. van Roy F and Berx G: The cell-cell adhesion molecule E-cadherin. Cell Mol Life Sci 65: 3756-3788, 2008.

11. Repetto O, De Paoli P, De Re V, Canzonieri V and Cannizzaro R: Levels of soluble E-cadherin in breast, gastric, and colorectal cancers. Biomed Res Int 2014: 408047, 2014.

12. Canel M, Serrels A, Frame MC and Brunton VG: E-cadherinintegrin crosstalk in cancer invasion and metastasis. J Cell Sci 126: 393-401, 2013.

13. Schmalhofer O, Brabletz S and Brabletz T: E-cadherin, betacatenin, and ZEB1 in malignant progression of cancer. Cancer Metastasis Rev 28: 151-166, 2009.

14. Place RF, Li LC, Pookot D, Noonan EJ and Dahiya R: MicroRNA 373 induces expression of genes with complementary promoter sequences. Proc Natl Acad Sci USA 105: 1608-1613, 2008.
15. Sun X, Rogoff HA and Li CJ: Asymmetric RNA duplexes mediate RNA interference in mammalian cells. Nat Biotechnol 26: 1379-1382, 2008.

16. Sano M, Sierant M, Miyagishi M, Nakanishi M, Takagi Y and Sutou S: Effect of asymmetric terminal structures of short RNA duplexes on the RNA interference activity and strand selection. Nucleic Acids Res 36: 5812-5821, 2008.

17. Morris KV, Santoso S, Turner AM, Pastori C and Hawkins PG: Bidirectional transcription directs both transcriptional gene activation and suppression in human cells. PLoS Genet 4: e1000258, 2008.

18. Schwartz JC, Younger ST, Nguyen NB, Hardy DB, Monia BP, Corey DR and Janowski BA: Antisense transcripts are targets for activating small RNAs. Nat Struct Mol Biol 15: 842-848, 2008.

19. Hu J, Chen Z, Xia D, Wu J, Xu H and Ye ZQ: Promoter-associated small double-stranded RNA interacts with heterogeneous nuclear ribonucleoprotein A2/B1 to induce transcriptional activation. Biochem J 447: 407-416, 2012.

20. Portnoy V, Huang V, Place RF and Li LC: Small RNA and transcriptional upregulation. Wiley Interdiscip Rev RNA 2: 748-760, 2011.

21. Morris KV, Chan SW, Jacobsen SE and Looney DJ: Small interfering RNA-induced transcriptional gene silencing in human cells. Science 305: 1289-1292, 2004.

22. Guo D, Barry L, Lin SS, Huang V and Li LC: RNAa in action: From the exception to the norm. RNA Biol 11: 1221-1225, 2014.

23. Nakagawa H, Hikiba Y, Hirata Y, Font-Burgada J, Sakamoto K, Hayakawa Y, Taniguchi K, Umemura A, Kinoshita H, Sakitani K, et al: Loss of liver E-cadherin induces sclerosing cholangitis and promotes carcinogenesis. Proc Natl Acad Sci USA 111: 1090-1095, 2014.

24. Corso G, Carvalho J, Marrelli D, Vindigni C, Carvalho B Seruca R, Roviello F and Oliveira C: Somatic mutations and deletions of the E-cadherin gene predict poor survival of patients with gastric cancer. J Clin Oncol 31: 868-875, 2013.

25. Zhang H, Stephens LC and Kumar R: Metastasis tumor antigen family proteins during breast cancer progression and metastasis in a reliable mouse model for human breast cancer. Clin Cancer Res 12: 1479-1486, 2006.

26. Yue X, Schwartz JC, Chu Y, Younger ST, Gagnon KT, Elbashir S, Janowski BA and Corey DR: Transcriptional regulation by small RNAs at sequences downstream from 3' gene termini. Nat Chem Biol 6: 621-629, 2010.

27. Jiao AL and Slack FJ: RNA-mediated gene activation. Epigenetics 9: 27-36, 2014.

28. Li LC: Chromatin remodeling by the small RNA machinery in mammalian cells. Epigenetics 9: 45-52, 2014.

29. Portnoy V, Lin SH, Li KH, Burlingame A, Hu ZH, Li H and Li LC: saRNA-guided Ago2 targets the RITA complex to promoters to stimulate transcription. Cell Res 26: 320-335, 2016.

30. Meng X, Jiang Q, Chang N, Wang X, Liu C, Xiong J, Cao H and Liang Z: Small activating RNA binds to the genomic target site in a seed-region-dependent manner. Nucleic Acids Res 44: 2274-2282, 2016.

31. Janowski BA, Huffman KE, Schwartz JC, Ram R, Nordsell R, Shames DS, Minna JD and Corey DR: Involvement of AGO1 and AGO2 in mammalian transcriptional silencing. Nat Struct Mol Biol 13: 787-792, 2006.

32. Kim DH, Saetrom P, Snøve O Jr and Rossi JJ: MicroRNAdirected transcriptional gene silencing in mammalian cells. Proc Natl Acad Sci USA 105: 16230-16235, 2008.

33. Morris KV: RNA-directed transcriptional gene silencing and activation in human cells. Oligonucleotides 19: 299-306, 2009.

34. Gagnon KT, Li L, Chu Y, Janowski BA and Corey DR: RNAi factors are present and active in human cell nuclei. Cell Rep 6: 211-221, 2014.

35. Chang CI, Yoo JW, Hong SW, Lee SE, Kang HS, Sun X, Rogoff HA, Ban C, Kim S, Li CJ, et al: Asymmetric shorterduplex siRNA structures trigger efficient gene silencing with reduced nonspecific effects. Mol Ther 17: 725-732, 2009. 